\title{
Electronic structure of disordered conjugated
}

\section{polymers: Polythiophenes}

\author{
Nenad Vukmirović, ${ }^{*}$ and Lin-Wang Wang \\ Computational Research Division, Lawrence Berkeley National Laboratory, Berkeley, CA 94720, \\ USA. \\ E-mail: NVukmirovic@lbl.gov
}




\begin{abstract}
Electronic structure of disordered semiconducting conjugated polymers was studied. Atomic structure was found from a classical molecular dynamics simulation and the charge patching method was used to calculate the electronic structure with the accuracy similar to the one of density functional theory in local density approximation. The total density of states, the local density of states at different points in the system and the wavefunctions of several states around the gap were calculated in the case of poly(3-hexylthiophene) (P3HT) and polythiophene (PT) systems to gain insight into the origin of disorder in the system, the degree of carrier localization and the role of chain interactions. The results indicated that disorder in the electronic structure of alkyl substituted polythiophenes comes from disorder in the conformation of individual chains, while in the case of polythiophene there is an additional contribution due to disorder in the electronic coupling between the chains. Each of the first several wavefunctions in the conduction and valence band of P3HT is localized over several rings of a single chain. It was shown that the localization can be caused in principle both by ring torsions and chain bending, however the effect of ring torsions is much stronger. PT wavefunctions are more complicated due to larger interchain electronic coupling and are not necessarily localized on a single chain.
\end{abstract}

Keywords: charge patching, localization, amorphous, density of states, molecular dynamics 
Nenad Vukmirović et al. Electronic structure of disordered conjugated polymers: Polythiophenes

\section{Introduction}

Semiconducting conjugated polymers ${ }^{1-9}$ are abundant organic materials that can be processed from solutions by inexpensive techniques, while exhibiting relatively high carrier mobilities. These features have led to their applications in electrical and optical devices such as light-emitting diodes, 3,4 solar cells, ${ }^{5,6}$ and field-effect transistors. ${ }^{7}$

It is currently understood that thin films of these polymers contain both ordered (crystalline) and disordered (amorphous) regions. ${ }^{10}$ Carriers in the crystalline regions are delocalized and therefore highly mobile, while carrier states in the amorphous regions are more localized. Consequently, electrical transport through the whole structure is largely influenced by the presence of amorphous regions. It is therefore very important to understand the nature of electronic states in the amorphous region. However, the polymer morphology in the amorphous region is rather complex. It consists of chains that are both twisted and bent forming a spaghetti-like structure. Furthermore, side chains are often attached to the main polymer backbone to make the polymer soluble. Due to such complexity of the material structure, there is a number of questions that naturally arise and need to be properly addressed. What is the cause of carrier localization? What is the degree of carrier localization? How does the chain morphology affect the electronic structure?

As a representative system for our study, we take polythiophene polymers and their solution processable derivative poly(3-hexylthiophene). Structural formulas of three units of these polymers are shown in Scheme 1a and b, respectively. Theoretical studies based on density functional theory (DFT) of the electronic structure of polythiophene derivatives arranged in a crystalline structure have been recently performed, ${ }^{10,11}$ however much less is known about the electronic structure of the amorphous phase where periodicity is broken and much larger supercells are required in a calculation.

The approach we take to tackle the problem of the electronic structure of conjugated polymers is centered around our recently developed charge patching method ${ }^{12-15}$ for electronic structure of organic systems ${ }^{16}$ which enables studies of systems with more than ten thousand atoms with the accuracy similar to the one of DFT in local density approximation (LDA). Atomic structure is 
Nenad Vukmirović et al. Electronic structure of disordered conjugated polymers: Polythiophenes

found from a classical molecular dynamics (MD) simulation.

\section{Theoretical and computational methodology}

\section{MD simulations of atomic structure}

Atomic structure of amorphous conjugated polymers is found from a classical molecular dynamics (MD) simulation with periodic boundary conditions. The following set of consecutive steps is performed in order to generate statistically probable atomic structures. $N_{c}$ separate chains each $N_{r}$ units long are initially randomly placed in a cubic box whose dimensions are several times larger than the dimensions of the box corresponding to the density of the system. A temperature of $1000 \mathrm{~K}$, significantly higher than the room temperature is initially imposed and the system is allowed to equilibrate for $200 \mathrm{ps}$ at that temperature. The size of the box is then gradually decreased during time $t$ to the value corresponding to the density of the natural system. After that, the temperature is decreased gradually down to $300 \mathrm{~K}$ during another time $t$. This step is followed by equilibration for $300 \mathrm{ps}$ at $300 \mathrm{~K}$ and a final relaxation to a local minimum. When $N_{c}=5$, we choose $t=200 \mathrm{ps}$ and when $N_{c}=20$ we take $t=1 \mathrm{~ns}$. The procedure described allows the system to explore a significant amount of configuration space, rather than being trapped in a local minimum which would highly depend on the random initial conditions. Similar MD based methods for generation of amorphous atomic structures have been applied to polyphenylene vinylene (PPV) oligomers, ${ }^{17}$ oligothiophenes, ${ }^{18}$ as well as other conjugated polymers. ${ }^{19,20}$

MD simulations were performed using the LAMMPS code. ${ }^{21,22}$ CFF91 class II force field ${ }^{23,24}$ was used to represent all interactions among atoms. Previous calculations indicated the existence of atomic structure rearrangements with important consequences for the electronic structure of the system. ${ }^{11}$ There is therefore a question whether the classical force field can take into account these effects and deliver the proper atomic structure. We have thus payed particular attention to this issue by comparing the force field calculations with quantum chemistry calculations, available in the literature for small systems. We have found that rotations of the bithiophene molecule around the 
Nenad Vukmirović et al. Electronic structure of disordered conjugated polymers: Polythiophenes

bond connecting the two rings are not well described by the original force field, i.e. the dependence of the energy on torsion angle does not reproduce well the calculations based on quantum chemistry methods. ${ }^{25}$ Since torsion angles determine the overall shape of the chain, this discrepancy would have a significant impact on the atomic and consequently electronic structure of the system. We have therefore corrected this discrepancy by replacing the terms describing this torsion in the original force field with a term that fits well the quantum chemistry calculations, as described in detail in Supporting Information. The use of interring torsional potential of bithiophene for the study of polythiophenes is justified by the fact that previous studies ${ }^{26,27}$ have indicated that these potentials are indeed very similar.

\section{Charge patching method for electronic structure}

After the atomic structure has been obtained, we proceed to calculate the electronic structure using the charge patching method. ${ }^{16}$ The details of the method have been previously presented ${ }^{16}$ and we only briefly outline it here. Charge patching method delivers a DFT/LDA quality charge density without actually doing a self-consistent DFT calculation. Charge density of a large system is obtained by adding together charge density motifs assigned to each of the atoms in the system. The motifs are generated from a DFT/LDA charge density (calculated using the code PEtot ${ }^{28}$ with norm conserving pseudopotentials with kinetic energy cutoff of $60 \mathrm{Ry}$ ) of small prototype systems in a manner previously described. ${ }^{16}$ Three unit long oligomers of thiophene (Scheme 1a) or hexylthiophene (Scheme 1b) were used as prototype systems for motif generation. The type assigned to each atom based on its bonds and nearest neighbors is shown in Scheme 1, while all atom types and all motifs of the two systems investigated are listed in Table $1 .{ }^{29}$

When the charge density is obtained, the single particle Hamiltonian is created by solving the Poisson equation for the Hartree potential and using the LDA formula for the exchange-correlation potential. The eigenstates can then be found using the folded spectrum method, ${ }^{30}$ as implemented in the ESCAN code, ${ }^{31}$ which is a method of choice when we are interested in only a few states around the gap. From the single particle Hamiltonian $H$, using the generalized moments method, ${ }^{32}$ 
Nenad Vukmirović et al. Electronic structure of disordered conjugated polymers: Polythiophenes

we also find the projected density of states defined as

$$
\rho_{\chi}(E)=\sum_{i}\left|\left\langle\psi_{i} \mid \chi\right\rangle\right|^{2} \delta\left(E-E_{i}\right)
$$

where $E_{i}$ are the eigenvalues and $\left|\psi_{i}\right\rangle$ the eigenstates of $H$. The function $\chi$ is chosen either as a random wavefunction (with an overall uniform distribution), or as a random function multiplied by a Gaussian. In the first case we get the information about the global density of states, while in the second case we get the information about local density of states. The number of generalized moments that we calculate corresponds to a spectral resolution of the order of $0.1 \mathrm{eV}$ in the energy domain. The truncation function in moments space ${ }^{32}$ corresponding to Gaussian broadening in energy domain was used to get smooth density of states profiles. It is important to note that the charge patching procedure scales linearly to the size of the system, ${ }^{16}$ as well as the folded spectrum method for a given number of states ${ }^{30}$ and the calculation of the density of states by the generalized moments method. ${ }^{32}$ Consequently, we can find several states in the spectral region around the gap, as well as the density of states in the whole spectral region for systems with even more than ten thousand atoms.

It is expected that charge patching method should work in systems with covalent bonds where the charge density around a given atom depends on its local environment only. The systems investigated here are exactly of this kind. We have previously shown ${ }^{16}$ that the method works well for a range of systems in ordered geometry, including polythiophenes, and for disordered alkane chains. This led us to expect that the method should work well for systems investigated here, too. To be fully certain about this we have performed an additional test for a system containing three 15 unit long oligomers of thiophene, a total of 321 atoms where it is still feasible to do a DFT calculation. The atomic structure of this test system was generated using the same MD-based procedure (with the box size proportionally smaller in this case) that was used for the systems that we investigated later. Therefore, the test system has a similar distribution of bending and torsion angles as the systems investigated and is fully suitable for testing the applicability of the charge patching pro- 
Nenad Vukmirović et al. Electronic structure of disordered conjugated polymers: Polythiophenes

cedure. The eigenstates obtained from the DFT/LDA calculation (using the code PEtot ${ }^{28}$ ) have been compared with those obtained from charge patching. The average error of eigenenergies was $20 \mathrm{meV}$ only, confirming our expectation.

\section{Results and discussion}

\section{P3HT}

We start the presentation of our results with the case of P3HT. A system containing $N_{c}=5 \mathrm{P} 3 \mathrm{HT}$ chains, each containing $N_{r}=20$ thiophene rings was investigated. This system contains 2510 atoms alltogether. The initial size of the box for MD simulation was $74 \AA$, while the final size was $29.286 \AA$, which corresponds to the experimental density of $1.1 \mathrm{~g} / \mathrm{cm}^{3} .{ }^{33-36}$ At the beginning of the simulation, all main chains were in all trans configuration with hexyl side chains distributed in the head-to-tail (Scheme 1b) arrangement. The final atomic structure obtained is presented in Figure 1a and the density of states of this system is presented in Figure 2a. Since the aim here is to describe the properties of infinite P3HT material, it should be also checked if the size of the box is large enough. For that purpose, another simulation was performed on a system containing $N_{c}=20$ chains, with a box which is proportionally larger compared to the previous case, whose final atomic structure is shown in Figure 1b. The density of states for this 10040 atom system is presented in Figure 2b. Since there is a close resemblance between the density of states of the two systems, a smaller 2510 atom system was chosen for the detailed study, later.

The calculated band gap of disordered P3HT is $1.50 \mathrm{eV}$. One should keep in mind that all calculations are based on LDA and therefore these gaps are susceptible to the well known LDA band gap problem. ${ }^{37} \mathrm{~A}$ direct comparison to experiment is therefore not possible. On the other hand, several experimental results ${ }^{38-40}$ indicate that both the energy gap measured in photoluminescence and absorption is larger in disordered than in ordered P3HT by about $\sim 0.25-0.5 \mathrm{eV}$. We have therefore also calculated the electronic structure of a two dimensional crystalline system consisting of periodically replicated infinite polythiophene chains. This kind of system has been 
Nenad Vukmirović et al. Electronic structure of disordered conjugated polymers: Polythiophenes

previously studied ${ }^{10}$ as a representative for electronic structure of crystalline alkyl-thiophenes. The spacing between chains was taken to be 3.8 Aand the angle of chain rotation about the chain axis of $30^{0}$ was assumed. ${ }^{10}$ The same bond lengths and angles as in the case of disordered P3HT were taken. The calculated band gap at the $\Gamma$ point is $1.25 \mathrm{eV}$ and the calculated indirect band gap is $1.12 \mathrm{eV}$. This result agrees with previous DFT calculations of two dimensional systems, ${ }^{10}$ and three dimensional crystalline alkyl-thiophene ${ }^{11}$ and thiophene ${ }^{41}$ polymers, where an indirect band gap, slightly smaller than the gap at the $\Gamma$ point, is obtained. We therefore find that the calculated difference between the gap of the crystalline and amorphous P3HT is consistent with experimental results reported in the literature. This result can be understood as a quantum confinement effect due to wavefunction localization as will be discussed below.

To gain understanding into the nature of the electronic structure of P3HT, we first probe the electronic structure locally, by calculating the local density of states at different points along the main chain, as well as on side chains. In the case of crystalline P3HT all centers of the rings would exhibit the same local density of state profile. This is no longer the case in disordered system and therefore local probing of the electronic structure gives an insight into a degree of disorder present in the system. A Gaussian with a standard deviation of 1.41 Åwas used as a local probe. In Figure 3 we present the results at five points on the same chain corresponding to the centers of rings 6,8 , 10, 12 and 14, which we label respectively as A, B, C, D and E.

A certain degree of fluctuations of the local bandstructure around the gap as one goes from point $\mathrm{A}$ to $\mathrm{E}$ along the main chain is evident from Figure 3. Several causes of these fluctuations, and therefore disorder in the system, are possible. First, it could be the interaction with other chains either via electronic coupling or via electrostatic interaction. Second, side chains of a given main chain also exhibit disordered configurations and electrostatic interaction with them can lead to spatially fluctuating potential along the main chain. Finally, the disorder in the conformation of the main chain itself is also a likely cause of these fluctuations. To distinguish between these three effects, we have performed several numerical experiments.

We have calculated the density of states along the same chain in the same geometrical arrange- 
Nenad Vukmirović et al. Electronic structure of disordered conjugated polymers: Polythiophenes

ment, but in the absence of other chains. The result of the calculation is shown for points B and D in Figure 3 with a thinner line. The difference between this and the previous calculation is obviously very small, so interchain interactions can be safely ruled out as the cause of local bandstructure fluctuations.

Next, we have analyzed the structure where we have kept all atoms of the main chains of P3HT in place, but side chains were removed and replaced by $\mathrm{H}$ atoms, thus transforming P3HT chains to PT chains. The comparison of the electronic structure of this structure with the one of P3HT presented in Figure 4 gives us information about the effect of side chains on the carrier states on the main chain. By comparing the global density of states in the spectral region around the gap (Figure 4e vs. f), as well as local density of states (Figure 4a vs. b and Figure 4c vs. d), we see only slight changes in the density of states. All main features of the electronic structure in this region remain unchanged. In the region further away from the gap (for example the region around $16 \mathrm{eV}$ in Figure 4) the density of states of the two structures obviously differs because this is the region where alkyl side chains start to contribute to the density of states.

From the previous analysis, it follows that the electronic structure of P3HT is mostly determined by conformations of individual chains. To gain insight into relation between the morphology of chains and the electronic structure, we have calculated several wavefunctions near the top of the valence band and the bottom of the conduction band. The valence band states are shown in Figure 5a and the conduction band states in Figure 5b. Each of these wavefunctions is localized on a single main chain, as a natural consequence of previous conclusions regarding weak interchain interactions. Additional confirmation that interchain interactions are weak comes from the comparison of HOMO and LUMO wavefunctions of a single chain in the absence of other chains, presented in Figure 5c, with the corresponding wavefunctions that are localized on the same chain in the whole P3HT system. The wavefunctions shown in Figure 5c are almost identical with the wavefunctions presented in same colors in Figure 5a and Figure 5b. Valence band states tend to be mostly localized in the regions of double intraring $C-C$ bonds, while conduction band states are localized in the region of $C-C$ interring bonds and single intraring $C-C$ bonds. Valence band 
Nenad Vukmirović et al. Electronic structure of disordered conjugated polymers: Polythiophenes

states therefore fully resemble the HOMO orbital of a straight PT chain, and conduction band states resemble the LUMO orbital of a straight PT chain. The disorder in the chain conformation comes from two morphological effects: rotation of the ring around the axis defined by interring bond (torsion) and rotation of ring around the axis perpendicular to interring bond (bending).

The effect of torsion is currently well understood. ${ }^{42}$ It decreases the overlap between the $\pi$ orbitals of neighboring rings and sufficiently large torsion breaks the extent of the wavefunction (conjugation length). Therefore it acts to increase the gap as a consequence of quantum confinement effect.

On the other hand, less is known about the effect of chain bending. To gain some qualitative insight, we have calculated the DFT/LDA electronic structure of the three ring thiophene chain for different bending angles between the end rings and the center ring. The dependence of the band gap on bending angle and the HOMO orbitals for bending angles of $15^{\circ}$ and $60^{\circ}$ are presented in Figure 6. In contrast to the effect of torsion, bending decreases the band gap. This is a consequence of the fact that bending increases the overlap of $\pi$ orbitals from neighboring rings. In a periodic structure with equal bending angle between neighboring rings this would increase the bandwidth and decrease the band gap. As can be seen from the wavefunctions in Figure 6 bending also tends to localize the wavefunction towards the bending region. To some extent, the effect of bending at one place in the chain can be understood as the defect that creates a localized state in the gap.

The average angle between the interring bond and the ring plane (bending angle) in our structure is equal to $7.8^{0}$ only. This indicates that the effect of chain bending on the electronic structure is weak, since much larger angles are required for a significant effect, as can be seen in Figure 6. Since in our structures, there are practically no bending angles larger than $15^{\circ}$, the difference between the gaps at $0^{0}$ and $15^{0}$ which is $45 \mathrm{meV}$ can be considered to be the estimate for the upper limit for the influence of bending on eigenenergies. Consequently, one expects that the electronic structure and wavefunction localization will be determined by chain torsions. It has been previously shown ${ }^{42}$ that torsion angles of about $\sim 40^{0}$ and more are required to have a pronounced effect of torsion on the electronic structure. To gain insight into the influence of torsion in our structure 
Nenad Vukmirović et al. Electronic structure of disordered conjugated polymers: Polythiophenes

we have counted the fraction $f$ of torsion angles between neighboring rings that differ by less than $40^{0}$ from the angles of $0^{0}$ or $180^{\circ}$. These values of torsion angles allow the wavefunction to be spread between neighboring rings. For the analyzed structure, $f=44 \%{ }^{43}$ indicating that there is a significant probability for the extension of the wavefunction over several neighboring rings. Indeed, closer visual inspection of the wavefunctions shown in Figure 5a and Figure 5b indicates that they are typically localized along 4-6 rings and that their extent is broken by ring torsions. The 4-6 unit chains have LDA band gap in the region $2.1-1.8 \mathrm{eV}^{16}$ which is similar to the local band gaps of P3HT (Figure 3), which additionally verifies the fact that the effect of chain bending is weak, as there is no additional band gap decrease due to chain bending.

\section{Polythiophene}

Further insight into the electronic structure of conjugated polymer systems can be gained by studying PT. The essential difference between PT and P3HT is that due to absence of side chains, one can expect that electronic coupling between main chains could play a more significant role in the case of PT. We have investigated a system of $N_{c}=5$ thiophene chains, each $N_{r}=20$ units long. The initial size of the box in MD simulation was 60 Åwhich was decreased during the simulation down to $21.365 \AA$, corresponding to PT density of $1.4 \mathrm{~g} / \mathrm{cm}^{3}$. ${ }^{18}$ The electronic density of states of this system is presented in Figure 7b, lower panel. Similar to the case of P3HT we have also calculated the density of states for the system with $N_{c}=20$ chains to verify that the same features in the electronic structure are obtained. The results of this calculation are also shown in Figure $7 \mathrm{~b}$, upper panel.

To understand the degree of interaction among different chains, we compare in Figure 8 the local density of states at several points along one thiophene chain in the presence and absence of other chains. Fluctuations in the local electronic structure as one moves along the chain are present in the case of PT, similar to the case of P3HT. The essential difference is however that there is now a significant difference between the local densities of states in the presence and absence of other chains, which is a clear signature of interaction among chains. Therefore, in contrast to 
Nenad Vukmirović et al. Electronic structure of disordered conjugated polymers: Polythiophenes

P3HT, where disorder comes solely from the conformations of the main chain, there is another component in PT as a consequence of interactions among chains. It has been previously inferred ${ }^{44}$ based on the fits to the mobility measurements that the energetic disorder is larger in $\mathrm{OC}_{1} \mathrm{C}_{10}$ compared to $\mathrm{OC}_{10} \mathrm{C}_{10}$ PPV polymers and a larger conformational and configurational freedom of $\mathrm{OC}_{1} \mathrm{C}_{10}$ was suggested as a likely cause of the effect. Our results indicate that in principle the disorder caused by interaction among chains is another component of the disorder which could explain an increased disorder in a polymer with shorter side chains.

To further understand the nature of disorder in the investigated PT system, we present in Figure $9 \mathrm{a}$ and Figure $9 \mathrm{~b}$ the wavefunctions of several first states in the conduction and last states in the valence band. Some of the presented wavefunctions are still localized on a single chain as in the case of P3HT, however quite a few of them spread over more than one chain (see for example Figure 9c) or over different parts of the same chain. The fraction $f$ (previously defined in the text) of torsion angles allowing the wavefunction to be extended over neighboring rings is equal to $55 \%$ in this case, being somewhat larger than in P3HT. ${ }^{43}$ This allows a slightly larger extent of the wavefunctions along a chain than in the case of P3HT. The HOMO and LUMO of a single chain in the absence of other chains are presented in Figure 9d. While the HOMO orbital still resembles the first valence band wavefunction of the whole PT system localized on that chain (shown in red in Figure 9a), the LUMO orbital is significantly different than the first of the conduction band wavefunctions of PT localized on the same chain (shown in light blue in Figure 9b). As can be seen from Figure 8, chain interactions act by overall broadening of the density of states profile and reduction of the local gaps. This is also reflected in the global gap as the calculated LDA band gap of PT is $1.25 \mathrm{eV}$ which is smaller than in P3HT. We verify that this difference does not come from chain bending as the average bending angle which we calculate to be $6.7^{0}$ is even slightly smaller than in P3HT. The effects of the delocalization of the wavefunctions over more than one chain, the local band gap reduction and the broadening of the density of states, all indicate that there is a significant degree of interchain electronic coupling in PT.

Since we have shown the importance of interactions among chains to the electronic structure of 
Nenad Vukmirović et al. Electronic structure of disordered conjugated polymers: Polythiophenes

PT, we would also like to verify the importance of chain conformations. Therefore, we present in Figure $7 \mathrm{a}$ the density of states of each of the individual chains in the absence of other chains. There are significant fluctuations in electronic structures of different chains, as evident from Figure 7a. This is an additional demonstration of the strong effect of chain conformation on the electronic structure and its disordered nature, both in PT and P3HT.

\section{Conclusion}

We have studied the electronic structure of polythiophene disordered conjugated polymers. Our findings indicate that in alkyl substituted thiophene polymers the electronic structure in the spectral region around the band gap is fully determined by the conformation of the main thiophene chains. We show that interactions with side chains or other main chains contribute only weakly to the disorder in the electronic structure of the main chain. The localization of carriers to the region of several chain units takes place due to the torsions of the rings rather than due to chain bending which is weak. Our results indicate to a certain extent that the density of states in the region around the gap of alkyl substituted polymers can be understood simply as a superposition of the densities of states of individual main chains and that large scale calculation employed here might not be therefore necessary. On the other hand, for transport calculations which are of high interest and which we intend to pursue in our future work, single chain calculation is not sufficient. This is because a single chain cannot carry steady state current and interchain hopping must be taken into account. On the other hand, in PT without side chains there are two components of disorder in the electronic structure. In addition to the conformation of the chain, the interaction with the other chains now plays a more significant role due to wavefunction coupling between different chains.

\section{Acknowledgement}

This work was supported by the DMS/BES/SC of the U.S. Department of Energy under Contract No. DE-AC02-05CH11231. It used the resources of National Energy Research Scientific Com- 
Nenad Vukmirović et al. Electronic structure of disordered conjugated polymers: Polythiophenes puting Center (NERSC).

\section{Supporting Information Available}

The details of the modification of the force field. This material is available free of charge via the Internet at http://pubs.acs.org. 
Nenad Vukmirović et al. Electronic structure of disordered conjugated polymers: Polythiophenes

\section{References}

(1) Heeger, A. J. Rev. Mod. Phys. 2001, 73, 681-700.

(2) Chiang, C. K.; Fincher, C. R.; Park, Y. W.; Heeger, A. J.; Shirakawa, H.; Louis, E. J.; Gau, S. C.; MacDiarmid, A. G. Phys. Rev. Lett. 1977, 39, 1098-1101.

(3) Burroughes, J. H.; Bradley, D. D. C.; Brown, A. R.; Marks, R. N.; Mackay, K.; Friend, R. H.; Burns, P. L.; Holmes, A. B. Nature 1990, 347, 539-541.

(4) Goh, C.; Kline, R. J.; McGehee, M. D.; Kadnikova, E. N.; Frechet, J. M. J. Appl. Phys. Lett. 2005, $86,122110$.

(5) Li, G.; Shrotriya, V.; Huang, J. S.; Yao, Y.; Moriarty, T.; Emery, K.; Yang, Y. Nature Mater. 2005, 4, 864-868.

(6) Günes, S.; Neugebauer, H.; Sacrifitci, N. S. Chem. Rev. 2007, 107, 1324-1338.

(7) Zaumseil, J.; Sirringhaus, H. Chem. Rev. 2007, 107, 1296-1323.

(8) Rohlfing, M.; Tiago, M. L.; Louie, S. G. Synth. Met. 2001, 116, 101-105.

(9) Briere, J.-F.; Cote, M. J. Phys. Chem. B 2004, 108, 3123-3129.

(10) Street, R. A.; Northrup, J. E.; Salleo, A. Phys. Rev. B 2005, 71, 165202.

(11) Northrup, J. E. Phys. Rev. B 2007, 76, 245202.

(12) Wang, L.-W. Phys. Rev. Lett. 2002, 88, 256402.

(13) Li, J.; Wang, L.-W. Phys. Rev. B 2005, 72, 125325.

(14) Li, J.; Wang, L.-W. Phys. Rev. B 2003, 67, 033102.

(15) Wang, L.-W. Phys. Rev. B 2002, 65, 153410.

(16) Vukmirović, N.; Wang, L.-W. J. Chem. Phys. 2008, 128, 121102. 
Nenad Vukmirović et al. Electronic structure of disordered conjugated polymers: Polythiophenes

(17) Yang, P.; Batista, E. R.; Tretiak, S.; Saxena, A.; Martin, R. L.; Smith, D. L. Phys. Rev. B 2007, 76, 241201.

(18) Zhang, G.; Pei, Y.; Ma, J.; Yin, K.; Chen, C.-L. J. Phys. Chem. B 2004, 108, 6988-6995.

(19) Zhang, G.; Ma, J.; Wen, J. J. Phys. Chem. B 2007, 111, 11670-11679.

(20) Yang, H.-C.; Hua, C.-Y.; Kuo, M.-Y.; Huang, Q.; C.-L.-Chen, Chem. Phys. Chem. 2004, 5, $373-381$.

(21) http://lammps.sandia.gov.

(22) Plimpton, S. J. J. Comp. Phys. 1995, 117, 1-19.

(23) Hwang, M. J.; Stockfisch, T. P.; Hagler, A. T. J. Am. Chem. Soc. 1994, 116, 2515-2525.

(24) Maple, J. R.; Hwang, M.-J.; Stockfisch, T. P.; Dinur, U.; Waldman, M.; Ewig, C. S.; Hagler, A. T. J. Comp. Chem. 1994, 15, 162-182.

(25) Aleman, C.; Julia, L. J. Phys. Chem. 1996, 100, 1524-1529.

(26) Belletête, M.; Di Césare, N.; Leclerc, M.; Durocher, G. Chem. Phys. Lett. 1996, 250, 31-39.

(27) Westenhoff, S.; Beenken, W. J. D.; Yartsev, A.; Greenham, N. C. J. Chem. Phys. 2006, 125, 154903.

(28) http://hpcrd.lbl.gov/ linwang/PEtot/PEtot.html.

(29) At the present stage, the motifs have been developed only for ideal bond lengths and angles (the same values of bond lengths and angles as in a previous work ${ }^{16}$ were used). Therefore, a slight readjustment of the atomic positions is necessary in order to use these motifs. We have done this by taking special care not to cause changes in the overall structure of the chain, nor to cause local chain bending.

(30) Wang, L.-W.; Zunger, A. J. Chem. Phys 1994, 100, 2394-2397. 
Nenad Vukmirović et al. Electronic structure of disordered conjugated polymers: Polythiophenes

(31) Canning, A.; Wang, L. W.; Williamson, A.; Zunger, A. J. Comp. Phys. 2000, 160, 29-41.

(32) Wang, L.-W. Phys. Rev. B 1994, 49, 10154-10158.

(33) Mardalen, J.; Samuelsen, E. J.; Gautun, O. R.; Carlsen, P. H. Solid State Comm, 1991, 77, $337-339$.

(34) Mardalen, J.; Samuelsen, E. J.; Gautun, O. R.; Carlsen, P. H. Synth. Met. 1992, 48, 363-380.

(35) Marchant, S.; Foot, P. J. S. Polymer 1997, 38, 1749-1751.

(36) Kim, S.-S.; Na, S.-I.; Jo, J.; Tae, G.; Kim, D.-Y. Adv. Mater. 2007, 19, 4410-4415.

(37) Seidl, A.; Görling, A.; Vogl, P.; Majewski, J. A.; Levy, M. Phys. Rev. B 1996, 53, 3764-3774.

(38) Brown, P. J.; Thomas, D. S.; Köhler, A.; Wilson, J. S.; Kim, J.-S.; Ramsdale, C. M.; Sirringhaus, H.; Friend, R. H. Phys. Rev. B 2003, 67, 064203.

(39) Korovyanko, O. J.; Österbacka, R.; Jiang, X. M.; Vardeny, Z. V.; Janssen, R. A. J. Phys. Rev. B 2001, 64, 235122.

(40) Chen, T.-A.; Wu, X.; Rieke, R. D. J. Am. Chem. Soc. 1995, 117, 233-244.

(41) Puschnig, P.; Ambrosch-Draxl, C. Synth. Met. 2001, 119, 245-246.

(42) Bredas, J. L.; Street, G. B.; Themans, B.; Andre, J. M. J. Chem. Phys. 1985, 83, 1323-1329.

(43) The number $f$ refers to the structure with $N_{c}=5$ and $N_{r}=20$. We have calculated it also for three other structures with $N_{c}=20$ and $N_{r}=20$, where different random initial conditions were used in their generation. The fluctuations of $f$ were several $\%$ only. This indicates that the specified value of $f$ is representative for the amorphous structures generated using the described procedure.

(44) Martens, H. C. F.; Blom, P. W. M.; Schoo, H. F. M. Phys. Rev. B 2000, 61, 7489-7493. 
Nenad Vukmirović et al. Electronic structure of disordered conjugated polymers: Polythiophenes

Table 1: The list of atom types and charge density motifs used for charge patching of PT and P3HT systems. A certain type is assigned to an atom in a way indicated in Scheme 1. In the notation used for motifs, the central atom of the motif is specified first, followed by its nearest neighbors. In the case of hydrogen central atom, next nearest neighbors have to be indicated as well.

\begin{tabular}{|l|l|l|}
\hline system & atom types & motifs \\
\hline PT & $C_{2}, C_{3}, C_{4}$, & $C_{2}-C_{3} C_{2} H, C_{3}-C_{3} C_{2} S, C_{2}-C_{4} C_{2} H$, \\
& $H, S$ & $C_{4}-C_{2} S H, S-C_{3} C_{3}, S-C_{4} C_{3}$, \\
& & $H-C_{2}-C_{3} C_{2}, H-C_{2}-C_{4} C_{2}$, \\
& & $H-C_{4}-C_{2} S$ \\
\hline P3HT & $C_{2}, C_{3}, C_{4}$, & $C_{5}-C_{6} C_{4} C_{2}, C_{2}-C_{5} C_{3} H, C_{3}-C_{3} C_{2} S$, \\
& $C_{5}, C_{6}, C_{7}$, & $C_{4}-C_{5} S H, C_{3}-C_{5} C_{3} S, C_{5}-C_{6} C_{3} C_{2}$, \\
& $H, S$ & $C_{4}-C_{2} S H, C_{2}-C_{5} C_{4} H, S-C_{3} C_{3}$, \\
& & $S-C_{4} C_{3}, C_{6}-C_{6} C_{5} H H, C_{6}-C_{6} C_{6} H H$, \\
& & $C_{7}-C_{6} H H H, C_{6}-C_{7} C_{6} H H$, \\
& & $H-C_{2}-C_{5} C_{3}, H-C_{4}-C_{5} S$, \\
& & $H-C_{2}-C_{5} C_{4}, H-C_{4}-C_{2} S$, \\
& & $H-C_{6}-C_{6} C_{5} H, H-C_{6}-C_{6} C_{6} H$, \\
& & $H-C_{7}-C_{6} H H, H-C_{6}-C_{7} C_{6} H$ \\
& &
\end{tabular}


Nenad Vukmirović et al. Electronic structure of disordered conjugated polymers: Polythiophenes

Scheme 1: Structural formulas of three unit long oligomers of a) PT and b) P3HT. The atom types used for classification of motifs in charge patching method are also indicated.

Figure 1: The atomic structure in the case of: a) P3HT with 2510 atoms, b) P3HT with 10040 atoms. Hydrogen atoms have been removed for clarity. Main chains are shown in black and side chains in gray.

Figure 2: The dependence of density of states (arbitrary units, logarithmic scale) on energy in the case of: a) P3HT with 2510 atoms, b) P3HT with 10040 atoms.

Figure 3: Local density of states at different points (logarithmic scale, arbitrary units) in the same chain of P3HT. The points A, B, C, D and E correspond to the centers of rings 6, 8, 10, 12 and 14 (of 20 unit long chain). Local density of states at points B and D in the absence of other chains is also shown in thinner lines for comparison.

Figure 4: Comparison of the density of states of $\mathrm{P} 3 \mathrm{HT}$ and the structure where side chains were replaced by $\mathrm{H}$ atoms.

Figure 5: Isosurface plots of P3HT wavefunctions. Isosurfaces correspond to the probability of finding an electron inside the surface of 50\%. Atomic structure of the main chains is also shown, while side chains have been removed from the figure for clarity.

a) First seven states in the valence band of P3HT. The calculated energies of the states are $18.910 \mathrm{eV}$ (dark blue), $18.888 \mathrm{eV}$ (green), $18.755 \mathrm{eV}$ (light blue), $18.690 \mathrm{eV}$ (red), $18.682 \mathrm{eV}$ (pink), $18.675 \mathrm{eV}$ (black), $18.654 \mathrm{eV}$ (gray).

b) First seven states in the conduction band of P3HT. The calculated energies of the states are $20.411 \mathrm{eV}$ (dark blue), $20.452 \mathrm{eV}$ (green), $20.486 \mathrm{eV}$ (light blue), $20.518 \mathrm{eV}$ (red), $20.546 \mathrm{eV}$ (pink), $20.591 \mathrm{eV}$ (black), $20.596 \mathrm{eV}$ (gray).

c) The HOMO (gray) and LUMO (pink) state of a P3HT chain in the absence of other chains.

Figure 6: The dependence of the LDA band gap of the three ring thiophene chain on bending angle. Isosurface plots of HOMO orbitals for bending angles of $15^{\circ}$ and $60^{\circ}$ are shown in insets.

Figure 7: Density of states of: a) the individual chains of PT in the absence of other chains; b) the PT systems containing 5 and 20 chains. 
Nenad Vukmirović et al. Electronic structure of disordered conjugated polymers: Polythiophenes

Figure 8: Local density of states at three points A, C, and E corresponding to the centers of the rings 6,10 and 14 of the same polythiophene chain in the presence of other chains (thick line, lower panels) and their absence (thin line, upper panels).

Figure 9: Isosurface plots of PT wavefunctions. Isosurfaces correspond to the probability of finding an electron inside the surface of $50 \%$. Atomic structure of the system is also shown.

a) The last seven states in the valence band of PT. The calculated energies of the states are $18.352 \mathrm{eV}$ (dark blue), $18.157 \mathrm{eV}$ (green), $18.082 \mathrm{eV}$ (light blue), $18.060 \mathrm{eV}$ (red), $17.998 \mathrm{eV}$ (pink), $17.950 \mathrm{eV}$ (black), $17.943 \mathrm{eV}$ (gray).

b) The first seven states in the conduction band of PT. The calculated energies of the states are $19.599 \mathrm{eV}$ (dark blue), $19.720 \mathrm{eV}$ (green), $19.740 \mathrm{eV}$ (red), $19.779 \mathrm{eV}$ (light blue), $19.800 \mathrm{eV}$ (pink), $19.839 \mathrm{eV}$ (black), $19.847 \mathrm{eV}$ (gray).

c) A closer look at the wavefunction with energy $18.157 \mathrm{eV}$. The two chains on which the wavefunction is mostly localized are shown in blue and red. The figure was rotated with respect to the others for clarity.

d) The HOMO (red) and LUMO (light blue) state of a PT chain in the absence of other chains. 
Nenad Vukmirović et al. Electronic structure of disordered conjugated polymers: Polythiophenes

a)

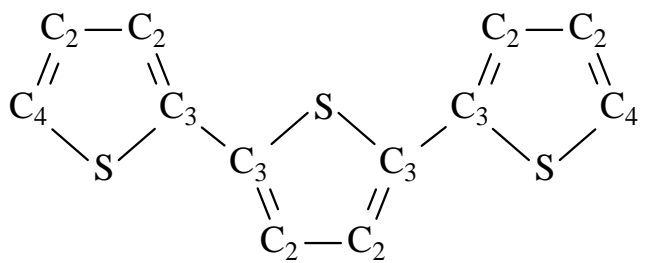

b)

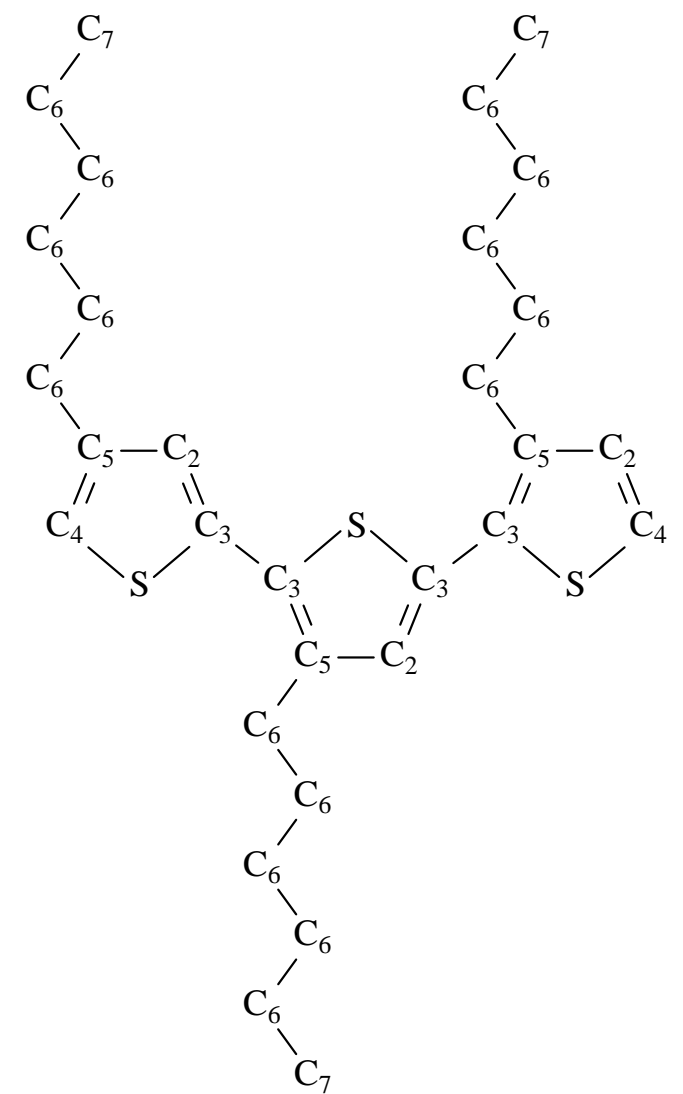

Scheme 1: 

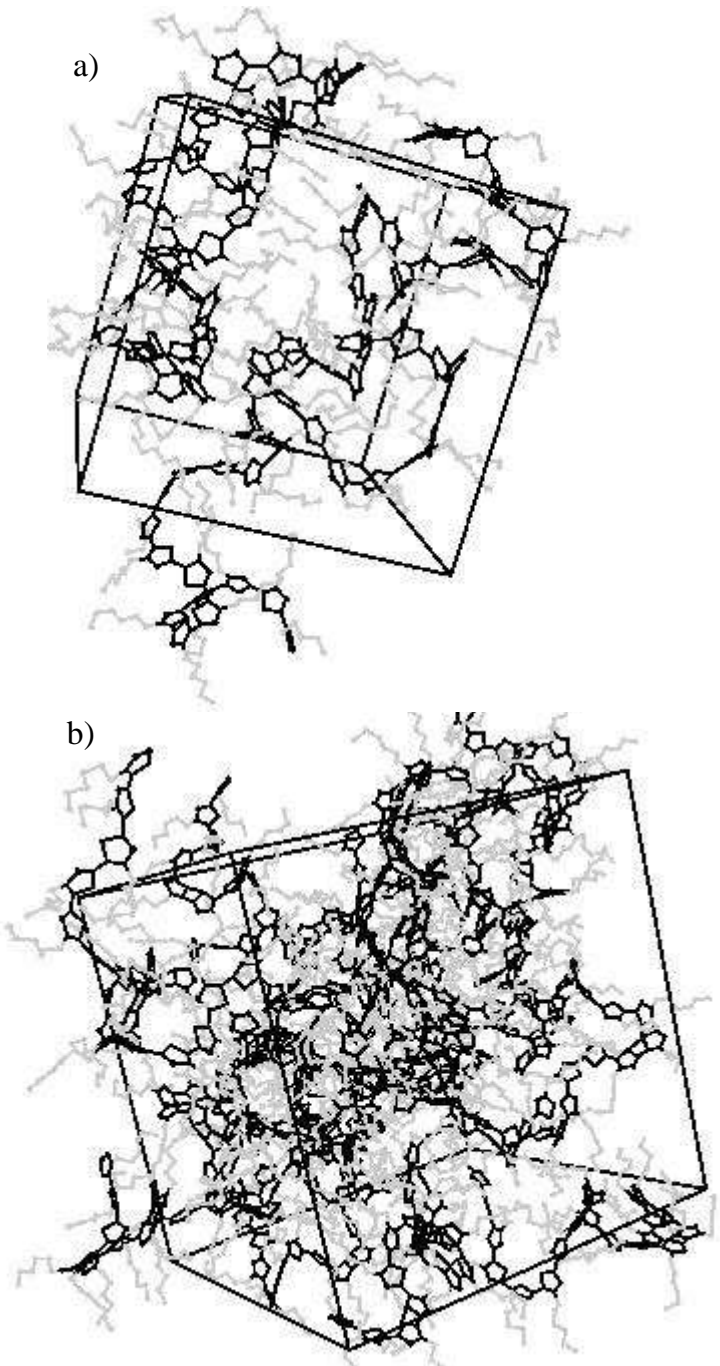

Figure 1: 
Nenad Vukmirović et al. Electronic structure of disordered conjugated polymers: Polythiophenes

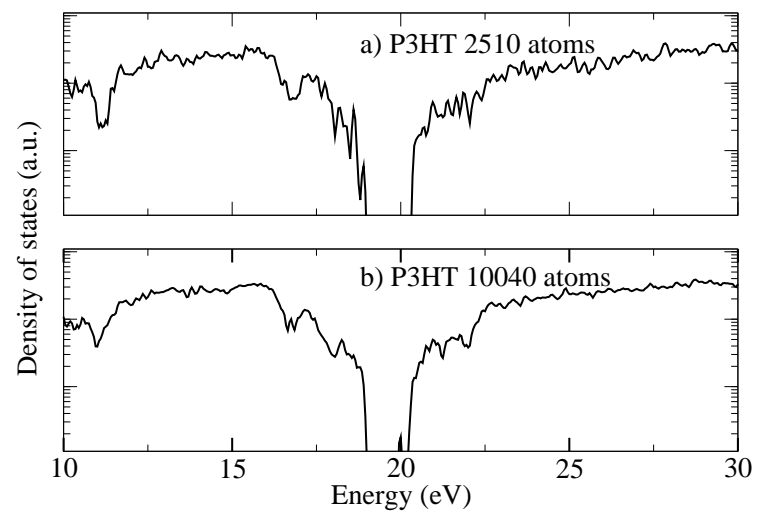

Figure 2: 


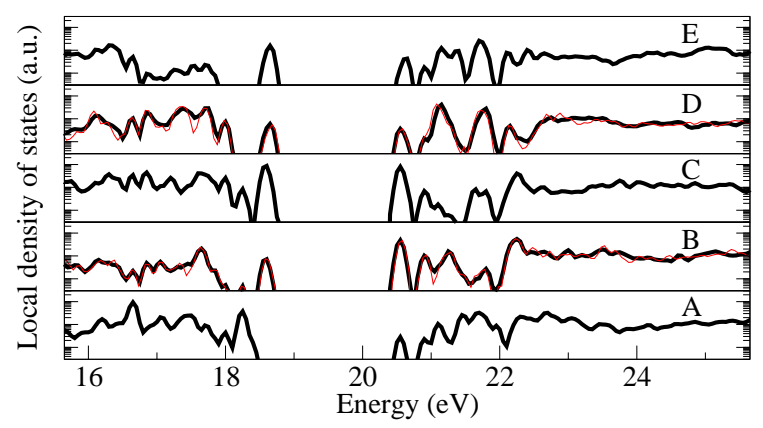

Figure 3: 
Nenad Vukmirović et al. Electronic structure of disordered conjugated polymers: Polythiophenes

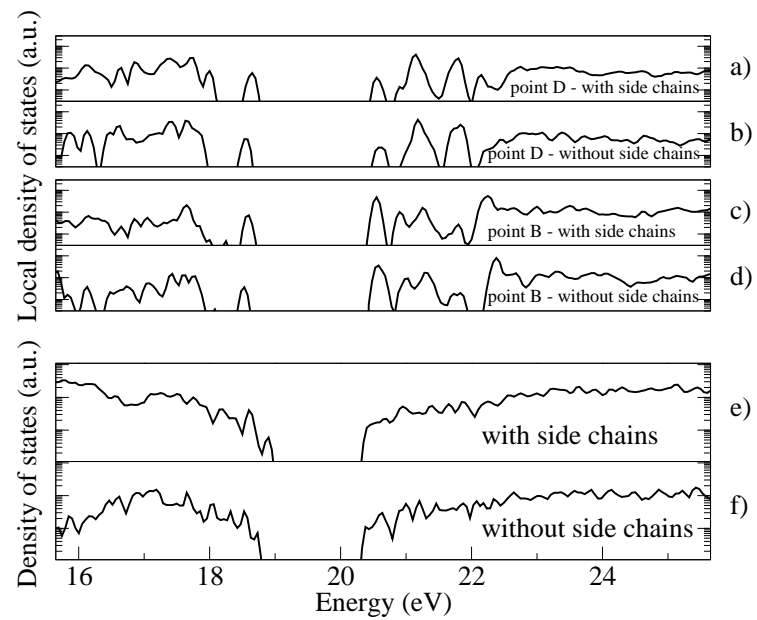

Figure 4: 

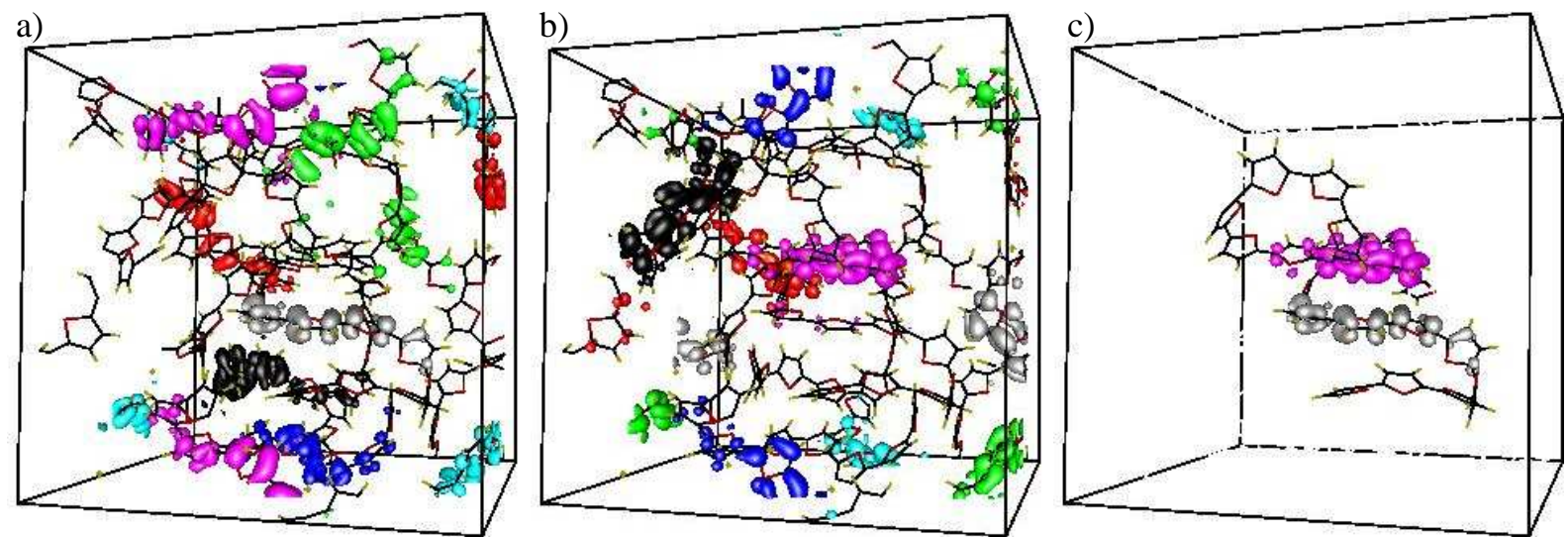

Figure 5: 
Nenad Vukmirović et al. Electronic structure of disordered conjugated polymers: Polythiophenes

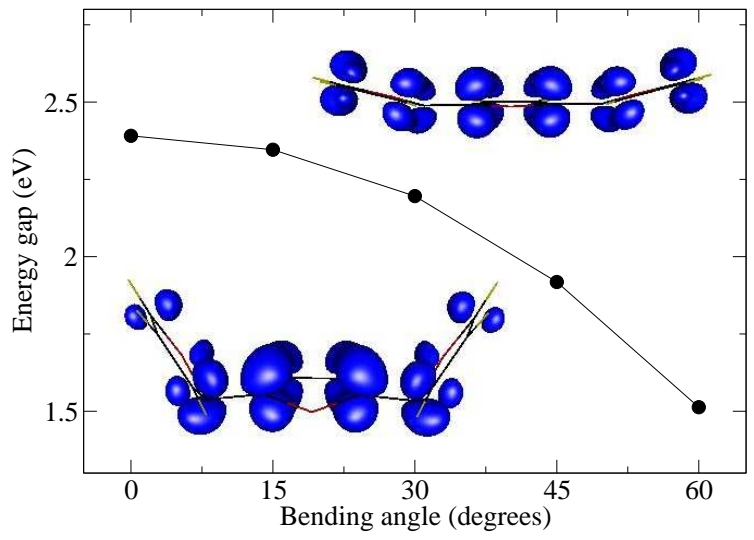

Figure 6: 
Nenad Vukmirović et al. Electronic structure of disordered conjugated polymers: Polythiophenes

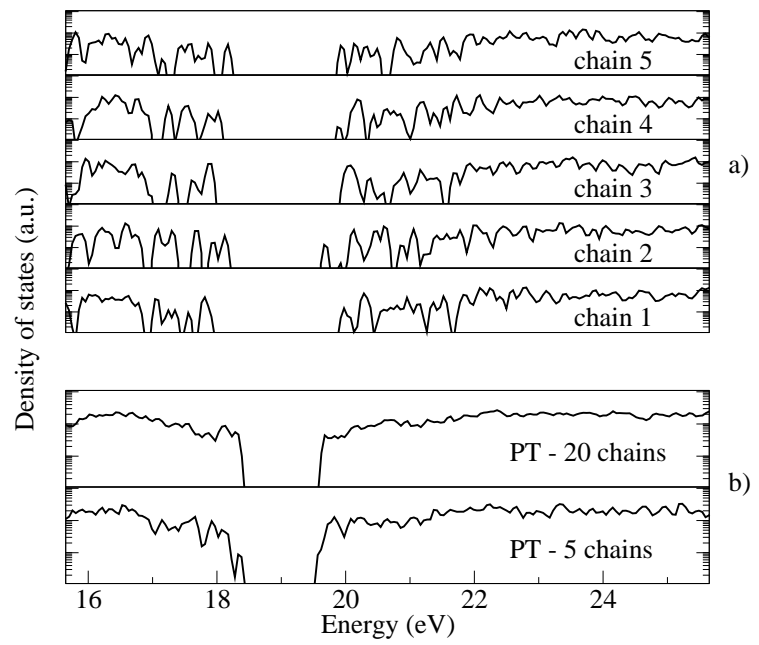

Figure 7: 
Nenad Vukmirović et al. Electronic structure of disordered conjugated polymers: Polythiophenes

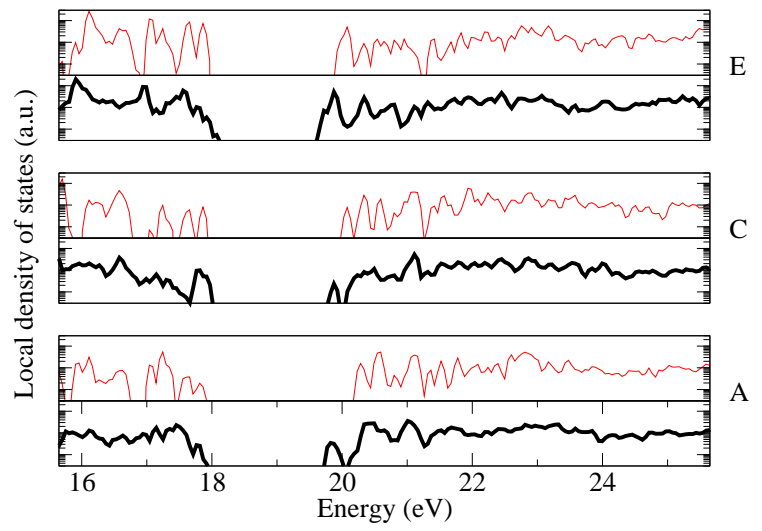

Figure 8: 
a)

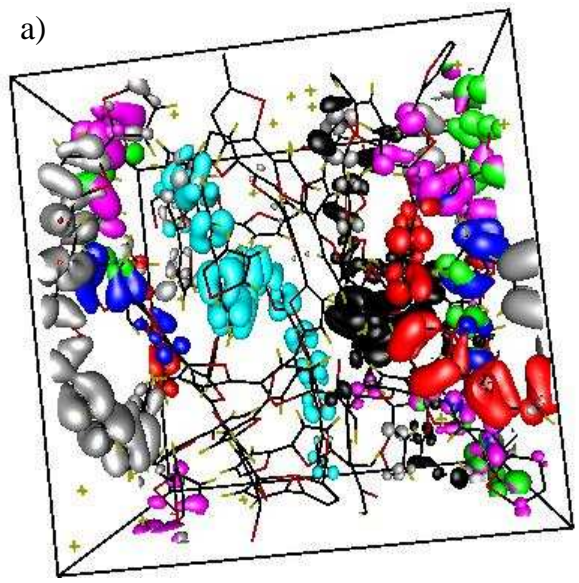

c)

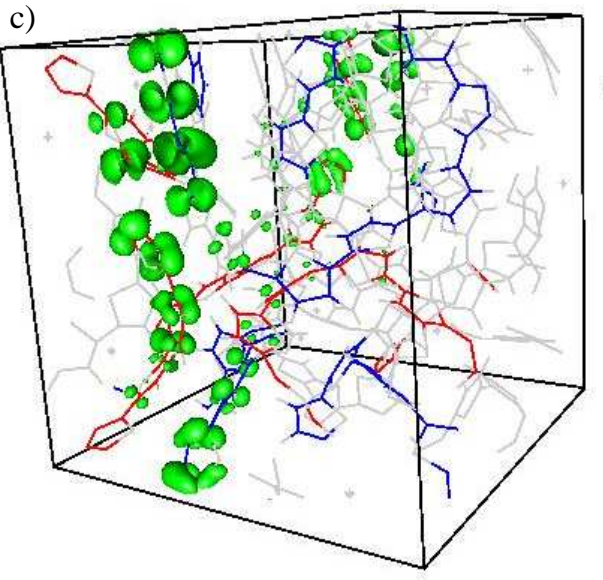

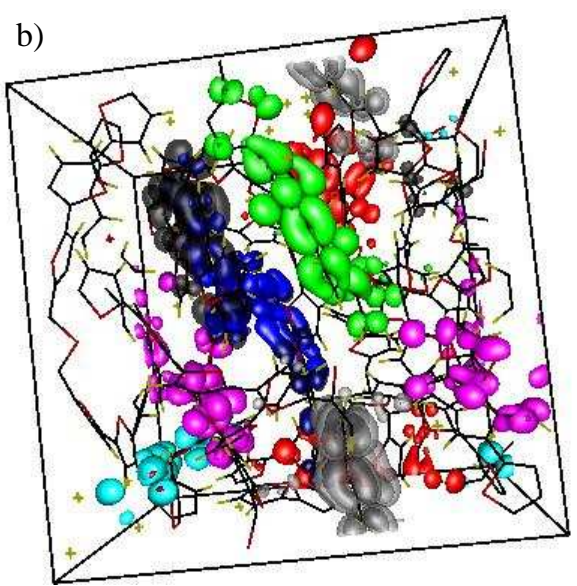

d)



Figure 9: 